\title{
Fractography of Dentin - Composite Interface Created at Positive Pulpal Pressure
}

\author{
V.M.Dusevich, J.H.Purk, J.D.Eick
}

University of Missouri - Kansas City, School of Dentistry, Kansas City, MO 64108

For proper bonding of resin composite restorations dentin should be etched to remove the smear layer and to create a layer of etched dentin, which after the infiltration with adhesive resin is called the hybrid layer. The mechanical properties of the dentin-composite interface (Fig. 1) are of immense importance to the durability of dental restorations, but it has been found that teeth restored under in-vivo conditions have lower microtensile bond strengths than teeth restored in the laboratory [1] because intrinsic fluid under positive pulpal pressure can permeate the dentin surface [2].

In this study, human teeth (with IRB approval) were restored a) without pulpal pressure and b) with artificial positive pulpal pressure of $5.0 \mathrm{~cm}$ water pressure to simulate a tooth anesthetized with epinephrine. Five adhesive systems from different manufacturers were used for restorations (Clearfil-SE, OneStep Plus, Peak-SE, Prompt L Pop and PQ1.) Twenty four hours after the restoration, all teeth were sectioned to obtain rectangular beam-shaped specimens with a cross section of $\sim 0.5 \mathrm{~mm}^{2}$ with no notch at the adhesive junction. Microtensile tests showed that for all adhesives but Clearfil-SE, the application of positive pulpal pressure reduced the bond strength [3]. The fractographic features of specimens treated with Clearfil-SE were similar regardless of whether the specimens were prepared under positive pressure or not (Fig. 2). The most common feature of specimens with low mechanical properties, treated under pressure with OneStep Plus, Peak-SE and PQ1, was a smooth fracture along the hybrid layer-adhesive interface (Fig. 3a). This fractogram is very similar to the image of a wet specimen of etched dentin (without adhesive application) as seen in an environmental SEM (Fig. 3b), with the exception of the presence of fractured resin tags in dental tubules in Fig. 3a. That similarity suggested minimal differences between the sample that was acid etched and treated with an application of adhesive compared to the sample whose dentin was only acid etched. The smoothness of the fracture surface suggested a very weak adhesion of the adhesive layer to hybrid layer. To check the integrity of the hybrid layer, ultrathin sections for TEM imaging were cut from the specimen shown in Fig. 3a (along with a specimen prepared without pulpal pressure). No embedding was performed, because electron densities of embedding resin and adhesive resin are approximately the same, so these resins could be undistinguishable in TEM. While the specimen treated without pulpal pressure had a well infiltrated hybrid layer and resin tags in tubules (Fig. 4a), the specimen treated with positive pressure had resin only in tubules and no hybrid layer, which suggests that no real infiltration occurred and that adhesive resin formed only a thin layer on the top of etched dentin layer. This conclusion was confirmed by observation of the specimen which had very low tensile strengths $(7.8 \mathrm{Mpa})$ and clearly demonstrated "smooth regions" formed by resin films detached from dentin surface (Fig. 5) Another common feature of excessively wet bonding was phase (resin-water) separation (Fig. 6)

References

[1] J.H.Purk et al., JADA 2004;135: 185-193

[2] F.R.Tay, D.H.Pashley, Dent Mater 2001;17: 296-308

[3] J.H.Purk et al., Am. J. of Dent, accepted for publication, 2009

[4] This project was supported in part by USPHS Grant K23-DE016324. 


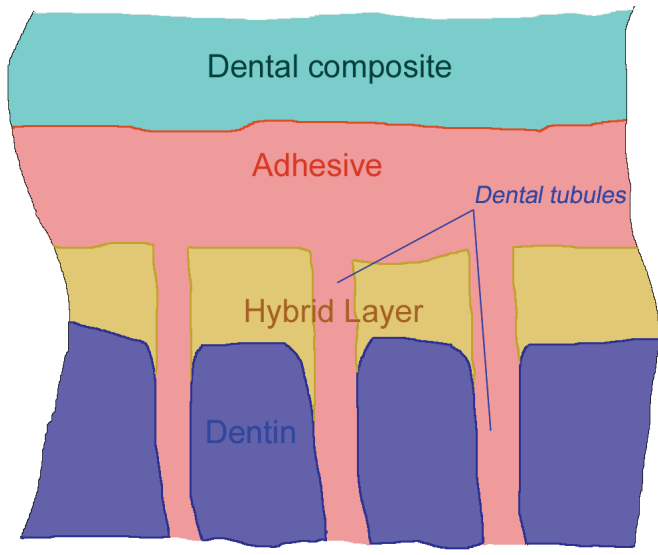

Figure 1. Dentin - composite interface
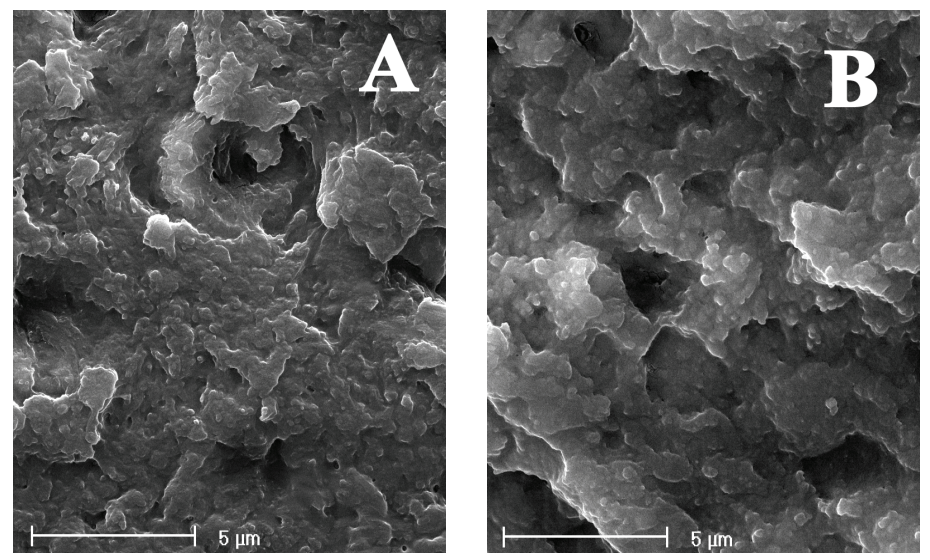

Figure 2. Fracture through hybrid layer of specimens treated with Clearfil-SE with (a) and without positive pulpal pressure (b)
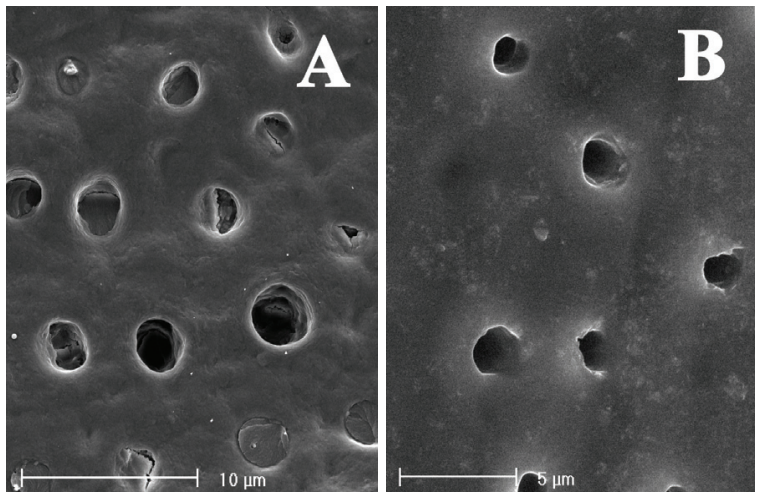

Figure 3. Smooth fracture surface of the specimen treated with OneStep Plus (a), and wet etched dentin specimen observed in ESEM mode (b)

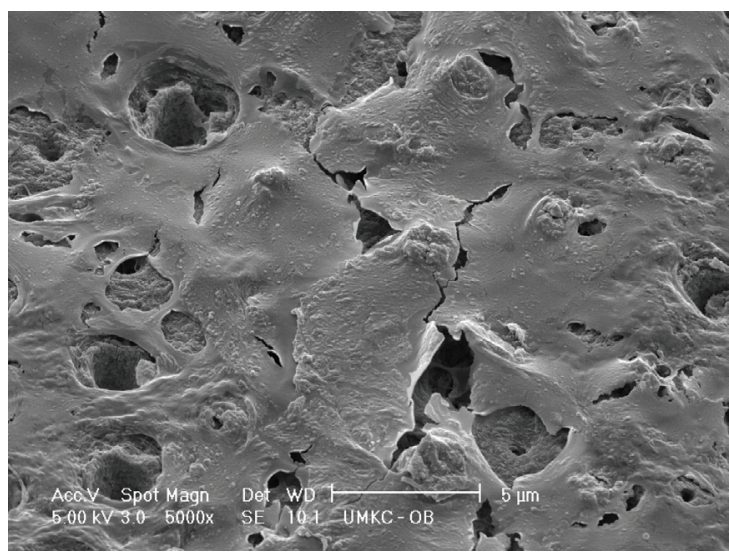

Figure 5. Specimen with very low tensile strength, treated with PQ1.
B
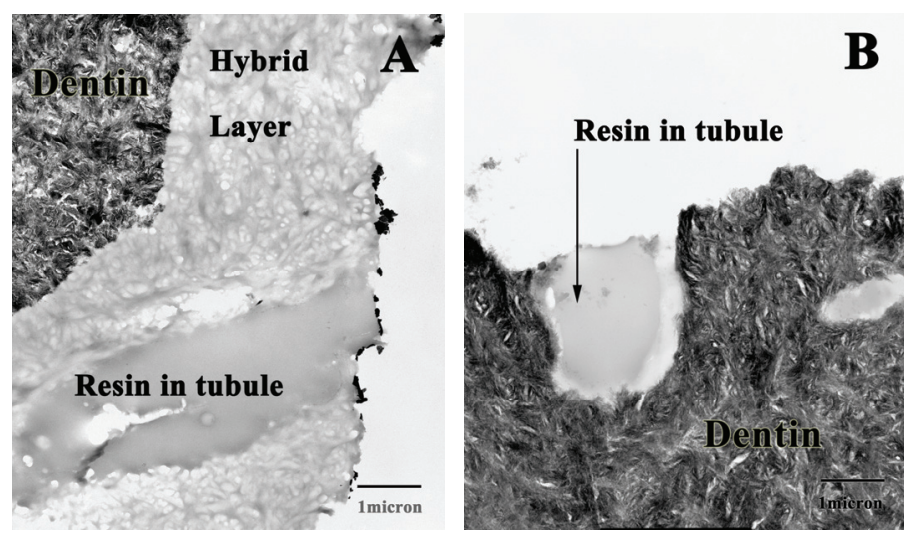

Figure 4. TEM micrographs of specimens treated with OneStep Plus without (a) and with positive pulpal pressure (b)

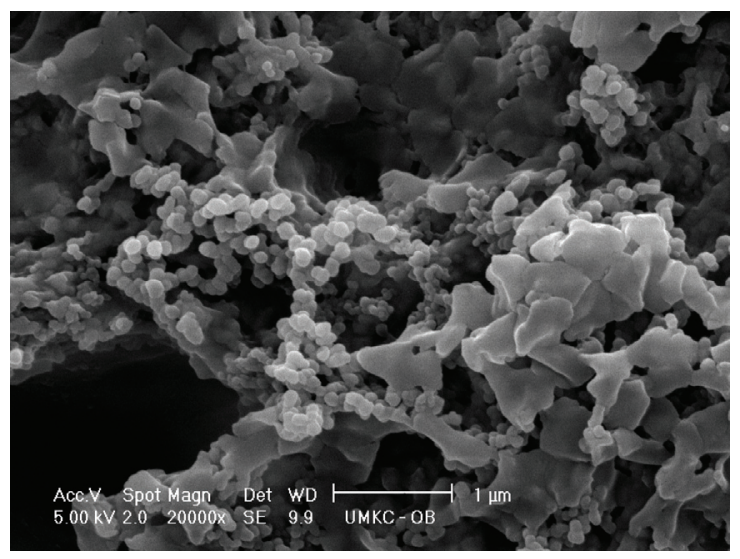

Figure 6. Phase separation in specimen treated with Prompt L Pop. 